$\xi=-1$

\title{
Towards a context-aware Wireless Sensor Networks
}

\author{
Zineb Aarab $^{1 *}$, Asmae El Ghazi ${ }^{1}$, Rajaa Saidi ${ }^{1,2}$, Moulay Driss Rahmani ${ }^{1}$ \\ ${ }^{1}$ LRIT, associated unit to CNRST (URAC 29) Faculty of sciences, Rabat IT Center, Mohammed V University in Rabat, Morocco. \\ ${ }^{2}$ SI2M laboratory, INSEA, BP 6217, Rabat, Morocco. \\ *Corresponding author E-mail: aarab.zineb@gmail.com
}

\begin{abstract}
Recently, the development of wireless sensor networks (WSNs) is spreading rapidly. WSNs are highly distributed self-organized systems which comprise a large number of resource constrained sensor nodes. Developers of WSNs face many challenges from communication, memory, limited energy... Also, mobility has become a major concern for WSN researchers. Indeed, Mobile WSNs (MWSN) consist of mobile sensor nodes that can move on their own and also interact with the physical environment. Developing applications for MWSN is a complicated process because of the wide variety of WSN applications and low-level implementation details. Integrating contextawareness can improve MWSN applications results. In this paper, some research issues and challenges involved in the design of WSNs are presented. Model-Driven Engineering offers an effective solution to WSN application developers by hiding the details of lower layers and raising the level of abstraction. In this sense, we propose a context-aware WSN architecture and WSN metamodel to ease the work for developers in this field.
\end{abstract}

Keywords: Wireless Sensor Network; Context; Context-Awareness; Mobility; Security.

\section{Introduction}

The tiny sensor nodes communicate within a short distances and collaboratively work towards fulfilling the application specific objectives of WSN's [1]. Recently context-awareness has been a great deal of interest. Using conventional methods of data gathering and processing in WSNs could lead to some of the problems, so the inclusion of context-awareness in WSNs can solve the problem to a great deal. Context is usually used to represent the information type in a system. The introduction of the contextawareness in the systems has helped to retrieve the information regarding the system rather easily. Context aware systems are able to adapt their operations to the current context without any user intervention and thus it increases the usability and effectiveness by taking environmental context into account. Context based information gathering for WSNs need to have proper information and measure of the context what we are using to represent system. In this paper we propose a model for Context-Aware WSNs, which shows how the context awareness is employed in WSN.

Applications for WSNs are varied, typically involving some kind of monitoring, tracking, or controlling. Specific applications include habitat monitoring, object tracking, nuclear reactor control, fire detection, and traffic monitoring. As WSNs, deal with real world environments sensor data must be delivered within time constraints so that appropriate observations can be made or actions taken. To date, the limited results that have emerged for WSN regarding real-time issues have been in routing. Many other functions must also meet real-time constraints including: data fusion, data transmission, target and event detection and classification, query processing, and security. It is important not only to develop real-time protocols for WSN, but associated analysis techniques must also be developed.

This research paper presents an overview of the notion of the context, WSN and MWNS and propose a context-aware WSN archi- tecture trying to meet with real time constraints to get better results for WSN applications.

The remaining of the paper is organized as follows: Section 2 presents the definition of the context. The difference between WSNs and MWSNs is presented in section 3. While section 4 describes the WSN metamodel. Section 5 shows our proposed context-aware WSN architecture. Finally, Section 6 concludes our work.

\section{Definition of the context}

For a formalization of context-aware routing, a definition of context and context-awareness is required at the first place.

More than 200 definitions were proposed about the term context thus the definition of what to consider as context varies highly across different fields. The word Context has given birth to a prominent research field which emerged in the early 90's. First "Context" derived from the Latin con (with or together) and texere (to weave). From the artificial intelligence perspective, this notion could have many definitions in the literature depending on its use. [2] defined context as "interrelated conditions in which an object exists or occurs" which means that the context has a bilateral relation between occurring conditions. Examples [3] and synonyms as "situation and environment" [3]-[7] was also used to define context. This had derived some researchers to propose a systemoriented definition of the context like [8] who claimed that "context can be considered to be everything that affects the computation except the explicit input and output" which means that the user is replaced by the application. We can observe that those definitions lacks of generality.

Trying to solve this problem Dey [9] proposed another definition, the context is "any information used to determine the situation of an entity". An entity could be a person, place, or object that is considered relevant to the interaction between a user and an appli- 
cation, including location, time, activities, and the preferences of each entity. In a similar sitting [10] tried to give an operational definition of the context by classifying the context into one of the following five categories:

- Individuality context includes properties and attributes defining the entity within the context (e.g. language, behaviour, preferences and goals of a learner, in the case that the learner is the entity [11]).

- Time context includes all temporal coordinates (e.g. points in times, long-term courses).

- Location context includes all spatial coordinates, for instance physical location, location in relation to people or resources, or virtual location (IP address).

- Activity context is concerned with all tasks, goals and actions the entity may be involved in goals, tasks and actions.

- Relations context captures information about any possible relation the entity may establish with another entity (social, functional and compositional relations) of example with the students, teachers and resources.

At the early age of the nineteens' most definitions were from a static view but [12] tried to formalize the context concept, from a dynamic perspective, by considering that the context cannot be described completely and is always relative to another context; and that the context has an infinite dimension [13].

Following this discussion about the static and dynamic aspects involved in the manipulation of context, [14] considered the two main aspects of context: context as a representational problem and context as an interactional problem. Then he claimed that the aspect that notices the interaction of objects is the correct one and not just the representational one.

Hence, it is challenging to define the word 'context' and many researchers tried to find their own definition for what context actually includes. For instance, in [15] we can say that this term is mostly adopted to indicate a set of attributes that characterize the capabilities of the access mechanism, the preferences of the user, and other aspects of the context in which information and services are delivered, these may include the access device (even in the presence of strong heterogeneity of the devices) [15].

Moreover, the critical importance of this concept has been the inspiration of several studies which gave multiple ramifications of its dimensions: spatial, spatial mobility, spatiotemporal, environment and personal dimension [16]. And so many efforts have been made since to categorize contexts: personal context which in cludes (physical and mental context); physical and conceptual context; physical and logical coordinate; while others consider state-based and event-based context as a categorization [17]. [18] categorized through tree main categories: (1) Physical environment context, it concerns the physical world (time, temperature, etc; (2) User context (preferences and needs...); (3) Virtual environment context where each component of the distributed system is aware of existing services.

[2] categorized context into three categories using a conceptual categorization-based technique on three main questions that can be used to determine the context [22]:

1) Where are you: all location related information such as GPS coordinates, common names (e.g. coffee shop, university, police), specific names (e.g. Canberra city police), specific addresses, user preferences (e.g. user's favorite coffee shop).

2) With whom are you: includes information about the presented people around the user?

3) What resources are near you: the information about resources available in the area where the user is located, such as machinery, smart objects, and utilities?

This categorization is also termed as the operational vs. conceptual context by [19]:

1) Operational categorization: Categorize context based on how they were acquired, modeled, and treated.

2) Conceptual categorization: Categorize context based on the meaning and conceptual relationships between the context.
Within the scope of this paper, the use of contextual information is not restricted to the interaction between users and applications, but the interaction among the devices within a mobile wireless sensor network. Take wireless sensor network as an example, the term context refers to the situation and the environment of the sensor nodes, which are objects in the terminology of the given general definition. The concrete context metrics of the sensor node can be, for example:

- Location

- Energy level

- Connectivity

- Sensed data

- Individual preferences

- Mobility

- Traffic rates

- Link quality

The description of a current context then at least consists of the description of relevant criteria as defined above, as well as the current context values for all these criteria. Additionally, it can also contain rules for correct interpretation of the combined context. We classify the context into three groups: local, link, and global context.

- Local context: local context includes local attributes of network nodes, such as location, mobility and residual energy.

- Global context: global context includes diverse attributes of the network, such as network topology and traffic conditions.

- Link context: link context includes various properties associated with wireless links, such as link quality and bandwidth.

- Due to the dynamic nature of mobile wireless sensor networks, it is expensive to obtain and maintain global contexts. Therefore, local and link context should be exploited efficiently to improve system performance. Context-aware means that an entity performs an action while taking into account its own current context and the context of those it is interacting with.

In the scope of wireless sensor networks, context-awareness refers to the context information (mentioned above) that should depend on the specific requirements of the application.

\section{WSNs and MWSNs}

Recently, mobility has become a significant area for (Wireless sensor network) WSN researchers community. Indeed, mobile wireless sensor networks (MWSNs) are a particular class of WSN in which mobility plays a key role in the execution of the application [20].

To understand MWSNs it important first to know the common assumptions regarding statically deployed WSNs, and second the changes that occurs when introducing mobile entities.

- Localization: Node position could be determined only during initialization in statically deployed network. Still, those mobile nodes must continually obtain their position as they pass through the detection area. Which demands more time and energy, besides the availability of a fast localization service.

- Dynamic Network Topology: Classical WSN routing protocols [21], that present how to send messages across the network to reach the destination, usually depend on routing tables or recent route histories. Otherwise in dynamic topologies, table data quickly become obsolete, and route discovery must be repeated at a significant cost in terms of power, time, and bandwidth. In this sense, there is an active research area dedicated to routing in mobile ad hoc networks (MANETs), and MWSN can benefit from [22].

- Power Consumption: Power consumption models are highly different between WSNs and MWSNs. Indeed, both types of networks involve a significant energy cost in terms of 
wireless communication and must be used effectively. Yet, mobility requires additional power, and mobile entities are often equipped with a much larger energy reserve or have self-charging capability that allows them to connect to the power grid to recharge their batteries.

- Network Sink: In centralized WSN applications, the sensor data is transmitted to a base station, to be processed using resource-intensive methods. Some MWSNs use mobile base stations, which pass through the sensed region to collect data, or their position to minimize the number of transmission hops for the sensor nodes. [20]

\section{The proposed WSN metamodel}

Wireless sensor networks (WSNs) are used to detect events and / or collect data in physical observation areas. They grew rapidly in their scale and complexity as their fields of application extend from environmental monitoring to precision farming, from perishable food transport to disaster response...

In this section we present a WSN metamodel (see Fig. 1) that summarize all cases of WSNs. Our WSN metamodel enables users to model WSN systems. Applications can be constrained by a set of rules, and models can be validated against these rules.

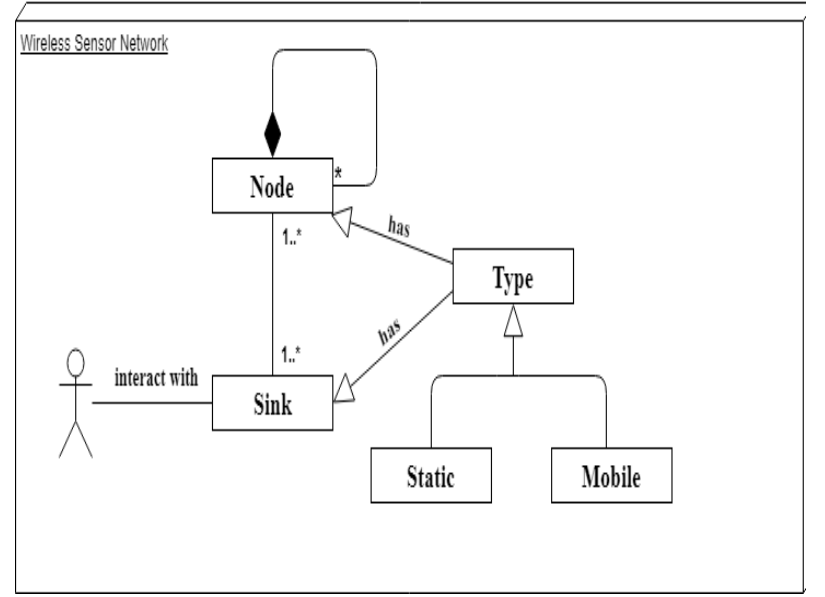

Fig. 1: The Proposed Wireless Sensor Network Met Model.

\section{- Node:}

The sensor node is one of the main parts of a WSN. The hardware of a sensor node generally includes four parts: the power and power management module, a sensor, a microcontroller, and a wireless transceiver. The power module offers the reliable power needed for the system. The sensor is the bond of a WSN node, which can obtain the environmental, and equipment status. A sensor is in charge of collecting and transforming the signals, such as light, vibration and chemical signals, into electrical signals and then transferring them to the microcontroller. The microcontroller receives the data from the sensor and processes the data accordingly. The Wireless Transceiver then transfers the data, so that the physical realization of communication can be achieved [23].

It is important that the design of the all parts of a WSN node consider the WSN node features of tiny size and limited power.

The use of the sensors node depends on the application, so the choice of the deployment strategy.

The node could be deployed randomly or not and could be mobile or static.

Depending on every detail on WSN the context change, that's why in this work we try to clarify this point and explain the WSN contexts:
The Sink or base station is the destination of the collected data and the link between sensors and the user. In WSN we can use one sink or multiple sink, a static sink or dynamic one by considering the circumstance with mean the WSN context-awareness:

- One Sink

- Multiple Sink

- Static Sinks

- Mobile Sinks

Different cases for nodes and sinks as presented and illustrated in the next section.

\section{Our context- aware WSN architecture}

The WSN are used in different applications, different constraints/ conditions are considered in the conception phase of the network. Herein, we present a context-aware WSN architecture (see Fig. 4) that resumes all possible cases, which would help WSN developers. The context-aware Manager (see Fig. 4) is the responsible of the decisions in real-time by analysing the sensed information and compare then to the normal cases (depending on the types of the application). We can distinguish different cases as follow:

1) Case 1: Static nodes and one static sink

This case is the standard case (see Fig. 2) where nodes are deployed randomly or not and communicate the information detected to the single base station, this structure caused some problems related to the routing, the reliability, and the communication delay [24].

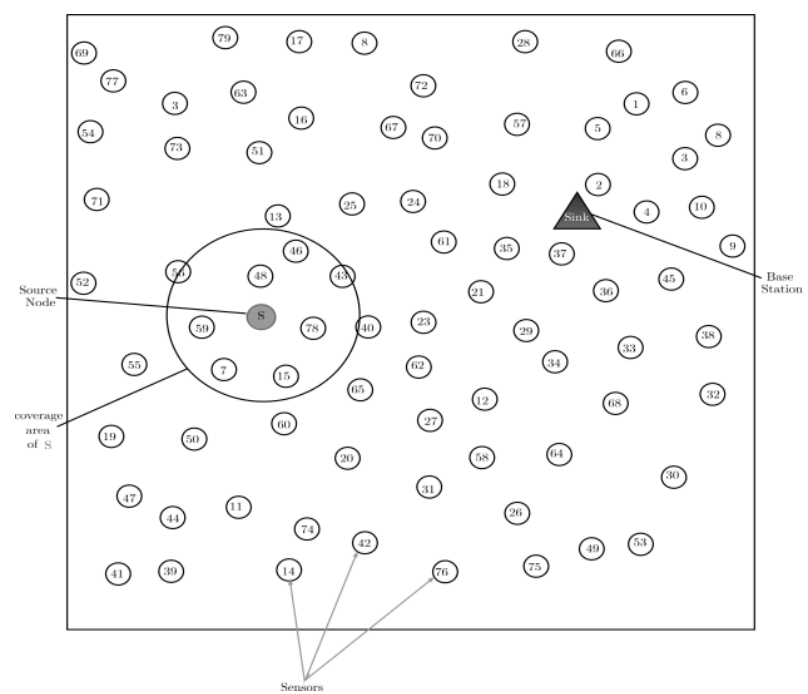

Fig. 2: Static Nodes and One Static Sink Illustration.

2) Case 2: Static nodes and one mobile sink

In this case some flexibility is added in the network, as the sink is mobile, so the communication of the data is somehow easier. This is the difference between this and the previous case but the problems need more changes and improvement in the WSN structure [25].

3) Case 3: Static nodes and multiple static sink

Multiple sink is a necessity for some application, where the user needs to keep in touch with the WSN and supervises the situation in real time and from different access point as illustrated in Fig. 3. The nodes in this case communicate the information with the nearest sink in order to assure a fast transmission of the data [26].

4) Case 4: Static nodes and multiple mobile sink

In the case of the multiple mobile sink the task for node is easier what mean that the user is informed in time about the event and the problems caused by the static sinks are fixed [27].

- Static nodes

- Mobile nodes

- Sink: 


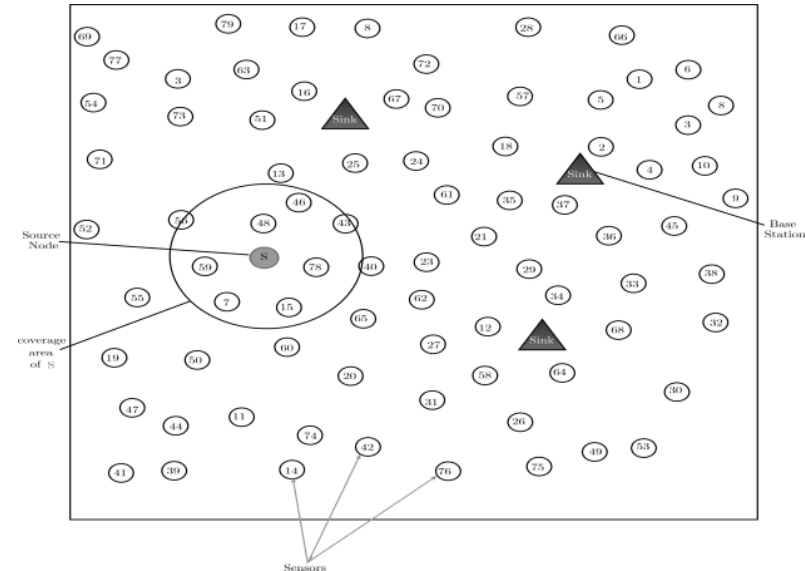

Fig. 3: Static Nodes and Multiple Static Sink Illustration.

5) Case 5: Mobile nodes and one static sink

The mobile WSN graves many advantages and assures a good coverage of the studied zone. The nodes change the position randomly or not depending on the application, thus the WSN is selforganised regularly and cover correctly the area to detect all events [28].

6) Case 6: Mobile nodes and multiple static sink

The applications that need mobile nodes in the network have some critical constraints, for example in medicine (the nodes move with the human body movements) as the health information is critical, multiple sink is a necessity in order to inform the doctor in time about the patient situation [29].

7) Case 7: Mobile nodes and multiple mobile Sink
Mobile sinks in this case facilitate the task in the applications and fixes some problems caused by the static case [30].

8) Case 8:other cases

There are many external conditions that could affect the WSN and its infrastructure, as the nature effects (wind, rain...) the animals or the area covered could be a dangerous place and damage the equipment... on the other hand there are virus or hackers that could affect the information transmitted.

Our proposed architecture takes in consideration all those cases and present an adaptive system (the context-aware manager) that accommodates with current situation and makes decisions according to the type of the applications or the random modifications made.

\section{Conclusion}

Context-awareness can solve some of the WSN problems, because its introduction into systems will help to retrieve system information easier. In this work, we propose a WSN metamodel and a context-aware WSN architecture that is able to adapt the system operations to the current context without any user intervention in order to increases the usability and effectiveness of WSNs by taking environmental context into account. We present a discussion of almost all possible cases for a WSN and demonstrate the advantage of our contribution.

For a future work, we will explore more details in the WSNs settings and constraints to offer more services and we want to propose the implementation of our proposed architecture.

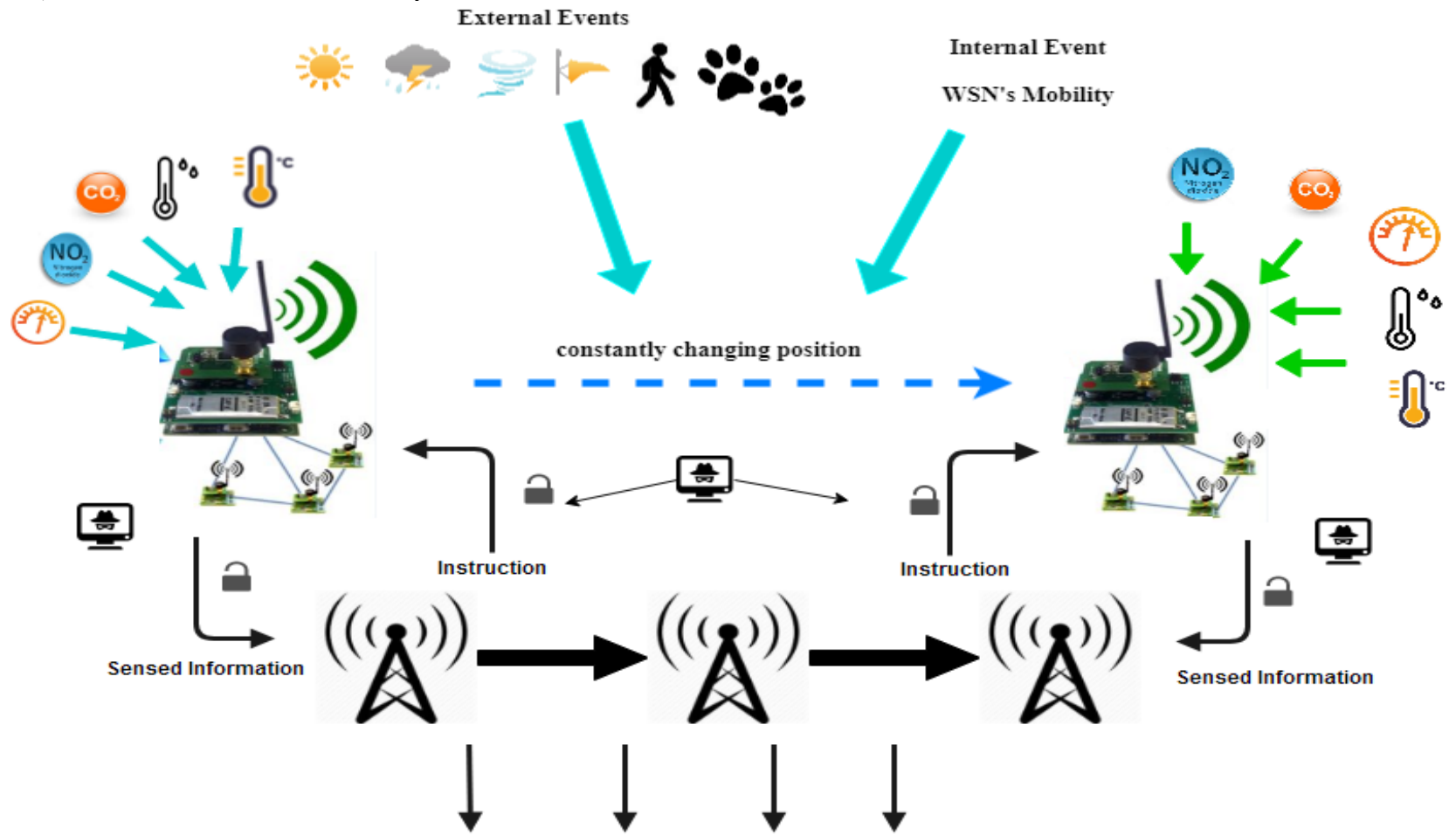

\section{Context-Aware Manager}

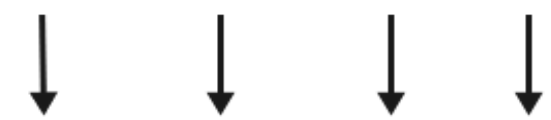

Send real-time information to the users

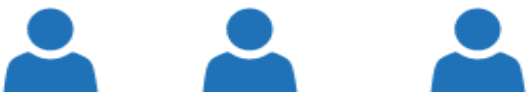

Fig 4: The Context-Aware WSN Architecture. 


\section{References}

[1] N. Xu, 'A survey of sensor network applications', IEEE communications magazine, vol. 40, no. 8, pp. 102-114, 2002. https://doi.org/10.1109/MCOM.2002.1024422.

[2] B. N. Schilit and M. M. Theimer, 'Disseminating active map information to mobile hosts', IEEE network, vol. 8, no. 5, pp. 22-32, 1994. https://doi.org/10.1109/65.313011.

[3] P. J. Brown, J. D. Bovey, and X. Chen, 'Context-aware applications: from the laboratory to the marketplace', IEEE personal communications, vol. 4, no. 5, pp. 58-64, 1997. https://doi.org/10.1109/98.626984.

[4] A. Ward, A. Jones, and A. Hopper, 'A new location technique for the active office', IEEE Personal communications, vol. 4, no. 5, pp. 42-47, 1997. https://doi.org/10.1109/98.626982.

[5] R. Hull, P. Neaves, and J. Bedford-Roberts, 'Towards situated computing', in Wearable Computers, 1997. Digest of Papers. First International Symposium on, 1997, pp. 146-153. https://doi.org/10.1109/ISWC.1997.629931.

[6] D. Franklin and J. Flaschbart, 'All gadget and no representation makes jack a dull environment', in Proceedings of the AAAI 1998 Spring Symposium on Intelligent Environments, 1998, pp. 155-160.

[7] T. Rodden, K. Cheverst, K. Davies, and A. Dix, 'Exploiting context in HCI design for mobile systems', in Workshop on human computer interaction with mobile devices, 1998, pp. 21-22.

[8] H. Lieberman and T. Selker, 'Out of context: Computer systems that adapt to, and learn from, context', IBM systems journal, vol. 39 no. 3.4 , pp. $617-632,2000$

[9] G. D. Abowd, A. K. Dey, P. J. Brown, N. Davies, M. Smith, and P. Steggles, 'Towards a better understanding of context and contextawareness', in Handheld and ubiquitous computing, 1999, pp. 304 307.

[10] A. Zimmermann, A. Lorenz, and R. Oppermann, 'An operational definition of context', in International and Interdisciplinary Conference on Modeling and Using Context, 2007, pp. 558-571. https://doi.org/10.1007/978-3-540-74255-5_42.

[11] S. B. Shum and R. Ferguson, 'Social learning analytics', Journal of educational technology \& society, vol. 15, no. 3, p. 3, 2012

[12] J. McCarthy, 'Notes on formalizing context', 1993.

[13] V. Vieira, P. Tedesco, and A. C. Salgado, 'Designing contextsensitive systems: An integrated approach', Expert Systems with Applications, vol. 38, no. 2, pp. 1119-1138, 2011. https://doi.org/10.1016/j.eswa.2010.05.006.

[14] P. Dourish, 'What we talk about when we talk about context', Personal and ubiquitous computing, vol. 8, no. 1, pp. 19-30, 2004 https://doi.org/10.1007/s00779-003-0253-8.

[15] M. Adorni et al., 'Reference architecture and framework', in Mobile Information Systems: Infrastructure and Design for Adaptivity and Flexibility, Springer Berlin Heidelberg, 2006. https://doi.org/10.1007/3-540-31008-8_2.

[16] B. Pernici and J. Krogstie, Mobile information systems. Springer, 2006. https://doi.org/10.1007/3-540-31008-8.

[17] C. Emmanouilidis, R.-A. Koutsiamanis, and A. Tasidou, 'Mobile guides: Taxonomy of architectures, context awareness, technologies and applications', Journal of Network and Computer Applications, vol. 36, no. 1, pp. 103-125, 2013. https://doi.org/10.1016/j.jnca.2012.04.007.

[18] S. Poslad, Ubiquitous computing: smart devices, environments and interactions. John Wiley \& Sons, 2011.

[19] A. H. Van Bunningen, L. Feng, and P. M. Apers, 'Context for ubiquitous data management', in Ubiquitous Data Management, 2005. UDM 2005. International Workshop on, 2005, pp. 17-24. https://doi.org/10.1109/UDM.2005.7.

[20] I. Amundson and X. D. Koutsoukos, 'A survey on localization for mobile wireless sensor networks', in Mobile entity localization and tracking in GPS-less environnments, Springer, 2009, pp. 235-254. https://doi.org/10.1007/978-3-642-04385-7_16.

[21] J. N. Al-Karaki and A. E. Kamal, 'Routing techniques in wireless sensor networks: a survey', IEEE wireless communications, vol. 11, no. 6, pp. 6-28, 2004. https://doi.org/10.1109/MWC.2004.1368893.

[22] M. Abolhasan, T. Wysocki, and E. Dutkiewicz, 'A review of routing protocols for mobile ad hoc networks', Ad hoc networks, vol. 2 , no. 1 , pp. 1-22, 2004. https://doi.org/10.1016/S15708705(03)00043-X.

[23] M. Healy, T. Newe, and E. Lewis, 'Wireless sensor node hardware: A review', in Sensors, 2008 IEEE, 2008, pp. 621-624.

[24] A. El Ghazi, B. Ahiod, and M. Abbad, 'TLBO-Based Routing Approach for Wireless Sensor Networks'.
[25] Y. Yun and Y. Xia, 'Maximizing the lifetime of wireless sensor networks with mobile sink in delay-tolerant applications, IEEE Transactions on mobile computing, vol. 9, no. 9, pp. 1308-1318, 2010. https://doi.org/10.1109/TMC.2010.76.

[26] T. K. Jain, D. S. Saini, and S. V. Bhooshan, 'Lifetime optimization of a multiple sink wireless sensor network through energy balancing', journal of Sensors, vol. 2015, 2015.

[27] S. Basagni, A. Carosi, C. Petrioli, and C. A. Phillips, 'Moving multiple sinks through wireless sensor networks for lifetime maximization', in Mobile Ad Hoc and Sensor Systems, 2008. MASS 2008. Fifth IEEE International Conference on, 2008, pp. 523-526. https://doi.org/10.1109/MAHSS.2008.4660067.

[28] A. El Ghazi, Z. Aarab, and B. Ahiod, 'Context-aware routing protocol based on PSO for mobile WSN', in 2017 3rd International Conference of Cloud Computing Technologies and Applications (CloudTech), 2017, pp. 1-6. https://doi.org/10.1109/CloudTech.2017.8284740.

[29] A. Erman-Tüysüz, 'Multi-sink mobile wireless sensor networks: dissemination protocols, design and evaluation', 2011.

[30] S. A. Munir, B. Ren, W. Jiao, B. Wang, D. Xie, and J. Ma, 'Mobile wireless sensor network: Architecture and enabling technologies for ubiquitous computing', in Advanced Information Networking and Applications Workshops, 2007, AINAW'07. 21st International Conference on, 2007, vol. 2, pp. 113-120. https://doi.org/10.1109/AINAW.2007.257. 